\title{
Psychodramatische Methoden in der Schreibwerkstatt
}

\author{
Carmen Mertlitsch
}

Online publiziert: 14. April 2016

(C) The Author(s) 2016. This article is available at SpringerLink with Open Access

Zusammenfassung Schreibwerkstatt und Psychodrama sind zwei sich gegenseitig befördernde Methoden. In diesem Beitrag wird aufgezeigt, wie sich diese beiden kreativen Ansätze ergänzen können und auf welche Art und Weise sich Psychodrama-Elemente in eine Schreibwerkstatt integrieren lassen. Einerseits lässt sich das Psychodrama erfolgreich einsetzen um tieferliegende Schreibprobleme zu erkennen und zu beheben, andererseits können dramatische Elemente in das Kreative Schreiben ebenso eingebaut werden wie Kreatives Schreiben in das Psychodrama. Einem theoretischen Überblick zur Verbindung Kreatives Schreiben, Schreibprobleme und Psychodrama folgt ein Werkstattbericht aus der Praxis der Autorin.

Schlüsselwörter Schreibwerkstatt $\cdot$ Kreatives Schreiben · Psychodrama ·

Schreibwissenschaft $\cdot$ Schreibprobleme $\cdot$ Schreibblockade $\cdot$ Schreibstörung

\section{Psychodramatic methods in the Writing Lab}

Abstract Writing Lab and Psychodrama are methods that complement each other well. The aim of this article is to show how to integrate psychodrama elements into a writing lab setting. On one hand psychodrama can be used to explore writing problems, on the other hand dramatic elements enhance the writing lab in a creative way. Conversely, creative writing can also be a productive method for therapeutic psychodramatic work. After a theoretical approach to the writing process, writing problems and creative writing is outlined, the author will give a workshop report based on her own work. Furthermore, she will discuss the benefits and limitations of combining these two creative methods.

C. Mertlitsch ( $\triangle)$

Alpen-Adria-Universität Klagenfurt, Universitätsstr. 65-67,

9020 Klagenfurt, Österreich

E-Mail: Carmen.Mertlitsch@aau.at 
Keywords Writing lab - Creative writing - Psychodrama - Writing science · Writing problems · Writing block · Writer's block · Writing disturbance

\section{Einleitung}

In abwechselnden Settings gestalte ich seit 2006 Einzel- und Gruppenschreibberatungen sowie Schreibwerkstätten für Studierende, Seniorstudierende und Lehrende sowie auch für Schulen und darüber hinaus auch Fortbildungsveranstaltungen für LehrerInnen. In jedem Semester halte ich eine Lehrveranstaltung zum Kreativen Schreiben ab mit verschiedenen Schwerpunkten wie Schreibtechniken, Lustvoll schreiben, Schreibprobleme überwinden, langgehegte eigene Schreibprojekte verwirklichen oder zu Textsorten wie Roman, Kinderbuch, Drehbuch und Fernsehserie. Meist finden die Lehrveranstaltungen 14-tägig nachmittags statt, es gibt zwischendurch aber auch Schreibwochen und atmosphärisch einzigartige und hochproduktive Schreibreisen, in denen neben den traditionellen Ansätzen der Schreibwerkstatt und der Schreibberatung auch psychodramatische Methoden zum Einsatz kommen. Psychodramatische Methoden eignen sich vor allem für die Bearbeitung prokrastinativen Verhaltens und anderer tieferliegender Schreibprobleme, sie können aber auch einfach nur die Schreiblust steigern oder die Bereitschaft erhöhen, über das eigene Schreiben nachzudenken. Ausgehend von den erstmals von Girgensohn (2007) vorgestellten Schreibreisen, habe ich das Methodenrepertoire um psychodramatische Arrangements erweitert. Es ist mir ein Anliegen, mit den solcherart gestalteten Schreibreisen und Schreibwerkstätten den Studierenden die Welt des Schreibens neu und schöpferisch zu eröffnen.

In diesem Artikel folgt nach einem sehr kurzen und selektiven Streifzug in die wissenschaftlichen Grundlagen der Schreibberatung und der Schreibwerkstatt ein Werkstattbericht, der von der diesjährigen Schreibwoche im Jänner sowie der Schreibreise im Juni 2015 in das Künstlerdorf Neumarkt an der Raab im österreichischen Burgenland berichtet. Es soll gezeigt werden, in welchen unterschiedlichen Formen sich psychodramatische Elemente in die Schreibwerkstattarbeit einbauen lassen.

\section{Schreibblockaden und Schreibstörungen}

Die Kunst einen Text zu schreiben ist eng verwoben mit dem Sich-AusdrückenKönnen. Zum Vorschein kommen dabei verschiedenartige Anteile der eigenen Persönlichkeit, Schreiben als Akt basiert eben auf der Ausdruckfähigkeit des oder der Schreibenden. Es geht, ebenso wie beim Psychodrama, darum, einen individuellen Weg zu finden die eigene Persönlichkeit zu entfalten. Es geht darum, sich zu zeigen, in Kontakt zu treten mit anderen und dabei die eigene Stimme zu finden, ihr eine Ausrichtung zu geben und eine Form. Der Unterschied zur Mündlichkeit besteht in einem Nicht-Mehr-Zurücknehmenkönnen der publizierten Texte, sei es als abgeschickter Brief, als E-Mail, als wissenschaftliche Arbeit, Kurzgeschichte, Roman, Gedicht oder Autobiographie. Mein Text, mein Schreiben, das bin ich. Darauf ha- 
be ich mich festgelegt. Und das, mit einem hohen Anspruch einhergehend, in einer möglichst zusammenhängenden, konsistenten und leserInnenfreundlichen Form.

Dieses Festschreiben der eigenen Vorstellungen, des Gemeinten, der zu vermittelnden Botschaft, macht ungeübten Schreibenden Angst und erzeugt oft Schreibhemmungen bis hin zu manifesten Schreibblockaden. Schreibprobleme haben mannigfache Ursachen, aber stets handelt es sich dabei um eine nicht vorhandene Fähigkeit, sich und seine Gedanken darzustellen. Das kann der Fall sein, wenn die Betroffenen noch unsicher darüber sind, wer sie eigentlich sind, was sie bewegt, welche Position sie im sozialen Miteinander einnehmen; ob (und wie) sie von anderen (und letztendlich von den LeserInnen) so angenommen werden, wie sie sind.

Schreiben kann entsprechend positive Gefühle wie Aufregung, Freude, Stolz oder ein Flow-Gefühl, aber auch negative Gefühle wie Unlust, Angst, Wut und Gefühle des Versagens mit sich bringen. Ein länger andauerndes, unbegleitetes Erleben negativer Gefühle wirkt sich hemmend auf einen zufriedenstellenden Schreibfortschritt aus. Es äußert sich in Schreibblockaden, abgebrochenen Texten und Aufschubverhalten (Prokrastination). Obwohl Schreibstörungen und Schreibprobleme fast allen Schreibenden bekannt sind und sie im Laufe eines Lebens immer wieder auftreten können, unterscheiden sie sich von tiefergehenden Schreibstörungen wie sie von Mike Rose (1984) oder Giselbert Keseling (2004) beschrieben werden. Letztere können persönlichkeitsbedingt sein, andererseits sind sie - wie die neuere Schreibforschung der letzten Jahrzehnte gezeigt hat (ein Überblick findet sich bei Girgensohn und Sennewald 2012) - immer auch situationsabhängig. Ein Zuviel an Vorgaben, (zu) hohe Erwartungen aus der Umgebung, vor allem von Seiten der Eltern und LehrerInnen sowie dann auch perfektionistische Erwartungen an sich selbst, unpräzise formulierte oder mündlich gegebene Schreibaufgaben, nicht-bewältigbar erscheinende Schreibhürden sowie fehlende Themeneingrenzung und fehlende Textsortenkenntnisse tragen zur Verfestigung der Problematik bei. Dazu kommen die Einsamkeit des Schreibens und unklares oder autoritär vorgetragenes negatives Feedback.

Neben der Schreibberatung ist die Schreibwerkstatt ein Ort, an dem die Betroffenen Unterstützung und Raum für eigenes Ausprobieren finden können.

\subsection{Kreatives Schreiben, Schreibwerkstatt und die Überwindung von Schreibproblemen}

Die Idee der Schreibwerkstatt stammt aus den methodischen Ansätzen des Creative Writing an US-amerikanischen Eliteuniversitäten zu Ende des 19. Jahrhunderts, wo das gemeinsame Schreiben zuerst für angehende LiteratInnen eingeführt wurde (Bräuer 1996). In den deutschen Sprachraum kam der Begriff des Kreativen Schreibens dann in den 1970er-Jahren, als erste literarische und themenbezogene Schreibwerkstätten eingerichtet wurden (Glindemann 2001). Von den Ansätzen der expressionistic rhetoric (z. B. Elbow 1973, 1981) ausgehend und später dann auch in Kombination mit genretheoretischen Ansätzen (ein Überblick findet sich bei Girgensohn und Sennewald 2012) ist es Ziel von Schreibwerkstätten, Schreibprobleme jeder Art zu überwinden und Schreiblust zu erfahren zu lassen, die TeilnehmerInnen den Schreibprozess bewusst erleb- und bewältigbar zu machen und sie mit den 
kommunikativen und stilistischen Anforderungen an Texten vertraut zu machen - in erster Linie durch das gegenseitige Voneinanderlernen in Peergroups.

Als Merkmale einer von mir moderierten Schreibwerkstatt gelten

1. Selbstbestimmung und Wertungsfreiheit,

2. der Vertrauensaufbau untereinander durch kollaborative Schreibspiele und gemeinsame Schreibaufgaben,

3. der offene Austausch über bisher gemachte Schreiberfahrungen,

4. das Kennenlernen von Schreibstrategien und -techniken sowie zwangloses Ausprobieren derselben,

5. individuelle Aufgabenstellungen für das Schreiben in und außerhalb der Gruppe, die einerseits anregende Vorgaben beinhalten (z.B. Themenauswahl oder Textsortenvorgaben, Materialvorgaben wie andere Texte, Postkarten, Bilder, Videomaterial, oder auch Vorgaben von Wörtern, in der Gruppe gemachte Wortschöpfungen, Silben-, Wörter- oder Strophenanzahl, Weglassen von Buchstaben im Text u. ä.), die die Schreibenden in ihrer Kreativität und Spontanität herausfordern, und die andererseits auch die Freiheit gewähren, von einer dieser Vorgaben abgesehen, den Text nach den eigenen Vorstellungen zu verfassen,

6. Analyse der eigenen, mitgebrachten Vorbildtexte,

7. gegenseitige Beratung sowie in anerkennendem Ton gehaltenes Textfeedback mit verschiedenartigen Feedbackmethoden - zuerst nur positiv, dann auch zunehmend kritisch-anregend,

8. Präsentation und Diskussion zumindest eines selbst eingebrachten Textes in der Werkstatt sowie

9. das Führen eines individuell gestaltbaren Schreibheftes, in dem die Texte gesammelt werden,

10. und die (schriftliche) Reflexionen über die eigene Schreibbiographie und den derzeitigen Schreibprozess enthalten (vgl. dazu auch Mertlitsch 2010, S. 45-55).

Auch Girgensohn (2007) wies darauf hin, wie wichtig bei Schreibenlernenden der soziale Faktor ist. Ein Lernerlebnis in der Gruppe bewirkt, dass Schreibende, so Girgensohn, nicht nur ihr Wissen darstellen, sondern auch in ihrer Gesamtpersönlichkeit gestärkt werden. Ihre kommunikativen Fähigkeiten werden ausgebaut und sie empfinden Freude beim Mitteinanderarbeiten und beim Schreiben.

Als Schreibberaterin geht es mir in den Schreibwerkstätten auch darum, den Schreibenden zu vermitteln, dass sie als Individuen eine einzigartige Sichtweise und - damit verbunden - einen individuellen sprachlichen Ausdruck haben. Durch die auf den Schreibentwicklungsstufen nach Bereiter (1980) aufbauenden Aufgabenstellungen, bei denen zuerst das expressive Schreiben (Elbow 1981) im Vordergrund steht, können sie beim regelmäßigen Schreiben ihre eigene Stimme (,voice“) finden. Sie erleben in diesem Prozess, dass sie etwas zu sagen haben, und dann beim Vorlesen, dass ihnen zugehört wird (vgl. auch Mertlitsch 2013). Nach der textuellen und sprachlichen Analyse von Vorbildtexten können sie ihre literalen bzw. literarischen Kompetenzen erweitern und diese Lernerfahrungen für das Schreiben anderer Textsorten nutzen. Wir reflektieren im Kurs auch den tiefergehende Zusammenhang von Schreiben und Emotion, auf den Kruse (1993, 2007, S. 61 f.) hingewiesen hat. Affektive Aspekte werden in der Schreibwerkstatt bewusst bearbeitet, wobei hier 
auch psychodramatische Techniken zum Einsatz kommen können. Vor allem, wenn es beim Schreiben in bestimmten Phasen wie beim Ideenfinden und während des eigentlichen Schreibvorgangs um Spontanität und Kreativität geht, lassen sich psychodramatischen Techniken einsetzen.

\subsection{Psychodrama in der Schreibwerkstatt}

Konkret eignet sich das Psychodrama als pädagogische Methode (vgl. Moreno 1959/2008, S. 88 und 106) für eine Schreibwerkstatt in sechsfacher Weise:

1. als Interaktions- und/oder Entspannungstechnik für die Arbeit mit Schreibgruppen, etwa in der Phase des Vertrauensaufbaus zum gegenseitigen Kennenlernen, nach einer längeren Schreibphase, um wieder in das Hier und Jetzt zu kommen, oder zwischen dem Textfeedback zur Entspannung nach dem konzentrierten Lesen, Zuhören und Überarbeiten (Stegreifspiel),

2. als Kreativitätstechnik zur Stofffindung in Form eines Stegreifspiels oder in schriftlicher Form als Dramolett, was sich gerade für das literarische Schreiben oder das gemeinsame Schreiben zu Beginn einer Schreibwerkstatt gut eignet,

3. für die Arbeit am Konzept eines Textes bzw. an der Textstruktur, sollte der Text schon in einer ersten Fassung vorliegen: Bei der Aufstellungsarbeit der Elemente erzählender und sachbezogener Texte kann der Erzählfluss bzw. die Linearität des Textes phantasievoll dargestellt werden (z. B. als ProtagonistInnenspiel oder in einer Weiterentwicklung als Textaufstellungsarbeit anhand eines Flussbildes oder einer Textwanderung),

4. für die Dramaturgie, die Arbeit am dramatischen Spannungsaufbau eines längeren (Prosa-)Textes wie eines Romans oder eines dramatischen Textes für Theater, Film und Fernsehen kann das Psychodrama - in Umkehrung des therapeutischen Ziels des Spannungsabbaus - auf eine möglichst dramatische Aufstellung der Konstellationen hinarbeiten. Die mitspielenden Personen können auf diese Art Konflikte konstruieren und sich besser zueinander in spannungsgeladene Beziehung setzen. Dank der herauszuarbeitenden Kontroversen können auch die künftigen LeserInnen/ZuseherInnen gefesselt werden - ähnlich wie es auch bei der Drehbuchstrukturaufstellung (DBSA) nach Varga von Kibed (2013) versucht wird (Situationsspiel, [dramaturgisches] Soziodrama),

5. zur Klärung und Integration der eigenen mit dem Schreiben einhergehenden Emotionen (ProtagonistInnenspiel),

6. zur Bearbeitung der oben beschriebenen tieferliegenden Schreibstörungen mit dem Ziel der Selbstverwirklichung (Monodrama, ProtagonistInnenspiel, auch hier eignet sich für den Einstieg das Dramolett, siehe unten).

Alle Instrumente und Techniken des Psychodramas wie Hilfs-Ich, der Rollentausch, das Doppeln oder Spiegeln können in diesem Zusammenhang zum Einsatz kommen (vgl. z. B. Soppa 2001/2004). Als alternativer Ansatz bietet sich gerade in einer Schreibwerkstatt ein verschriftlichtes Psychodrama an, z. B. in Form von selbst verfassten kleineren Dramen oder Dramoletten (siehe unten).

In einer einwöchigen Schreibwerkstatt im Jänner 2015 habe ich als Leiterin der Schreibwerkstatt einige psychodramatische Elemente in den Ablauf eingebaut, 
nämlich die Arrangements Dramolett, Stegreifspiel, dramaturgisches Soziodrama und Textaufstellungsarbeit. Diese psychodramatischen Arrangements erfüllen zwar wichtige psychosoziale und kreativitätsfördernde Aufgaben in der Schreibwerkstatt, müssen aber, was Ablauf und Ausführung betrifft, noch verfeinert werden. Im Juni 2015 gab es bei der Schreibreise die Möglichkeit, einen Schritt weiter zu gehen. In einer kleinen Gruppe von fünf Personen wurden tieferliegende Schreibstörungen bearbeitet und zwar in Form des ProtagonistInnenspiels. Ich habe dabei auch auf meinen bisherigen Erfahrungen in der individuellen Schreibberatung aufgebaut, bei der ich seit 2006 bedarfsorientiert das ProtagonistInnenspiel in Form des Monodramas bei der Darstellung der schreibbegleitenden Emotionen immer wieder einmal genutzt habe.

Im Folgenden möchte ich nun kurz drei der genannten psychodramatischen Einsatzmöglichkeiten näher darstellen.

\subsection{Arbeit mit Dramoletten}

Dramolette oder Minidramen sind kurze Einakter, die im Spiel bis zu 15 Minuten lang sind. In einer Schreibwerkstatt können die TeilnehmerInnen in einem Zeitraum von 15 bis 20 Minuten Dramolette verfassen, z. B. zu ihrem inneren Kritiker, dem zerstörerischen inneren Adressaten, mit dem sie hadern, aber auch als ein Gespräch mit dem Schreibwerkzeug (Bleistift, Schreibmaschine, PC oder i-pad) oder als Monolog des Textes, den sie schreiben sollen und der davon erzählt, wie es denn gerade um ihn bestellt sei oder der von der Motivation des Schreibenden berichtet (in Anlehnung an Ruhmann 1996, S. 218). Das Dramolett als verschriftlichte Form des Rollenspiels ermöglicht den Zugang zu sich selbst in aufrichtiger und manchmal auch ironisierender Form, sodass einerseits Berührtheit, andererseits auch eine gewisse innere Distanz zu den belastenden Situationen gefunden werden kann. In einem nächsten Schritt kann sich aus einem Dramolett ein tiefergehendes ProtagonistInnenspiel entwickeln, jedoch lässt sich ein Effekt meist schon durch den eigenen inneren Dialog sowie die expressive oder humorig überzogene Perspektive auf die jeweilige Schreibsituation gewinnen. Dramolette werden in Einzelarbeit, zu zweit oder in der Kleingruppe verfasst und dann als Lesedramen, als inszenierte Monodramen mit oder ohne Rollentausch oder als Gruppenspiel inszeniert. Hier das Beispiel von Richard dazu:

\section{Ich und mein Schreibproblem}

Problem (freundlich): Guten Morgen! Mir ist langweilig, willst du dich ein bisschen mit mir auseinandersetzen?

Ich (verschlafen): Nein! Bin nicht in der Stimmung!

Problem (schüttelt den Kopf): Du solltest wirklich etwas schreiben, du hast Abgabetermine.

Ich (genervt): Ja, gut. Dann eben. Du lässt mir ja doch keine Ruhe (beginnt zu schreiben).

Problem (schaut über die Schulter): Hmmmm ...

Ich: Was?? 
Problem: Du schreibst unzusammenhängenden Unsinn, der nichts mit der Aufgabe zu tun hat ...

Ich: Na und? Besser als gar nichts.

Problem: Schaust du ob noch Milch im Kühlschrank ist?

Ich: Gute Idee (Geht zum Kühlschrank und notiert was am Einkaufszettel).

Problem: So, das war doch schon recht produktiv, findest du nicht?

Ich (erstaunt): Echt? Wie jetzt?

Problem: Naja, du hast schon an deinem Text gearbeitet UND du hast dich um den Haushalt gekümmert. Zeit für ein wenig Unterhaltung.

Ich: Du hast recht, aber vielleicht sollte ich noch ein wenig ... weil ich habe nur noch so wenig Zeit und ...

Problem (hält sich die Ohren zu): Lalalalala, ich kann nichts hören.

Ich (brüllt): In zwei Tagen ist ABGABETERMIN!!!

Problem (erstaunt): Du hast doch schon mehr als genug Seiten geschrieben ...

Ich: Ja, aber ich bin vom Thema abgeschweift und habe den Text nicht korrekturgelesen, zu wenig Zitate drin und den Schlussteil noch gar nicht begonnen!

Problem (zuckt mit den Schultern): Ja, siehst du. Besser als gar nichts.

Ich: Ja, morgen ist auch noch ein Tag.

\section{Stegreifspiel in der Schreibwerkstatt}

Das Stegreifspiel eignet sich, wie oben dargestellt, besonders gut als Kreativitätssowie als Interaktions- und/oder Entspannungstechnik. Im Stegreifspiel könnte es z. B. darum gehen, dass die Mitspielenden verschiedene lesende und schreibende Berufe wie LiteratIn, JournalistIn, WerbetexterIn, ArchivarIn, KochbuchautorIn, WissenschaftlerIn oder BibliothekarIn verkörpern, die ihrem eigenen Temperament und ihrer Neigung entsprechen. Sie alle machen auf einem Boot oder in einem Bus eine gemeinsame Schreibreise und handeln dabei die für sie optimalen Schreibbedingungen aus. Es geht darum, im gemeinsamen Spiel herauszufinden, welcher Schreibtyp sie sind, welche Schreibstrategien sie verfolgen und wie die optimale Arbeitsumgebung strukturiert sein muss, so dass das Schreiben von möglichst angenehmen Gefühlen begleitet wird. Das Stegreifspiel kann auch völlig spontan und ohne Vorgaben von der Gruppe selbst gestaltet werden und als Stoffsammlung für eine spätere Schreibaufgabe dienen.

\section{Psychodrama bei Schreibblockaden und Schreibproblemen}

Während der Schreibreise wurden verschiedene Workshopangebote gestellt, u.a. eines zur psychodramatischen Bearbeitung manifestierter Schreibprobleme. Es meldeten sich fünf TeilnehmerInnen dazu an. Als geschlossene Gruppe suchten wir uns einen Raum für die Bühne auf dem Freiluftgelände und wir wurden auf der großzügigen Veranda eines alten Kinos fündig. Zum gegenseitigen Kennenlernen (der Workshop fand gleich am ersten Tag der Schreibreise statt) und zur Fokussierung auf das jeweilige Schreibproblem bot sich die Methode freewriting an. Sie ermöglichte 
ein direktes Inbeziehung-Setzen mit dem studentischen Schreiberleben der TeilnehmerInnen. Bei dieser Methode nach Elbow (1973, 1981; sowie Bräuer 2006) wird spontan, oft auch zu einem Thema wie mein Schreibproblem, darauflosgeschrieben ohne innezuhalten oder das bereits Geschriebene zu verbessern. Es geht darum, ohne innere Zensur sich in ein Thema hineinzuschreiben, die Gedanken dazu zu Papier zu bringen, ohne sie zu bewerten. Die Schreibenden sollten sich die Last von der Seele schreiben und damit eine schriftliche Reflexion voranbringen, ähnlich dem automatischen Schreiben. Das freewriting ist in Schreibwerkstätten das Mittel der ersten Wahl bei Schreibblockaden, weil jeder Text, der auf diese Weise zustande kommt, bereits einen Schreibfortschritt bedeutet und der Text zwar ein privater bleibt und nicht vorgelesen werden muss, jedoch als Grundlage für die nächsten Schritte dient.

Nach einem fünfzehnminütigen freewriting in der Erwärmungsphase wurde versucht, wie zu Beginn des Psychodramas üblich (Hollenstein-Burtscher 2004, S. 194 ff.), die Gruppe dialogisch im Hier und Jetzt zusammenzuführen und die TeilnehmerInnen in die Bereitschaft zu versetzen, ihre schwierigen und konflikthaften Situationen zu bearbeiten. Angesprochen wurden Versagensängste, die Angst vor der negativen Bewertung, das ständige Gefühl, etwas besser machen zu müssen oder nicht gut genug zu schreiben. Es ging um Schreibabbrüche und mangelnde Konzentration, die Vermeidungsverhalten nach sich zog. Der rege Austausch führte zur Vertrautheit und schließlich dazu, dass ein junger Mann mit Migrationshintergrund, F., ausgesucht wurde, sein prokrastinatives Verhalten, das sich mit der Zeit zu einer echten Schreibblockade entwickelt hatte, zu inszenieren.

F. richtete die Szene ein, suchte sich seine Mitspielerinnen, allesamt junge Frauen, aus, und kleidete sie ein. Sie übernahmen die Rolle des überwältigenden Schlafs (bis in den Nachmittag hinein), der Selbstdisziplin, des Widerstands und dann auch die der Mutter des jungen Mannes. Diese Anteile lagen miteinander in Konflikt und rangen um Vorherrschaft. Es stellte sich heraus, dass der Widerstand gegen das Schreiben eine Schutzfunktion in Hinblick auf das eigene Versagen und den massiven Druck aus dem Elternhaus einnahm. Die Szene wurde im psychodramatischen Verfahren durchgespielt und der Zusammenhang von äußerer Erwartung und mangelnder Selbstdisziplin wurde verdeutlicht. Dem Protagonisten, F., konnte man seine Fluchttendenzen auch im Spiel immer wieder anmerken. Er rang um Worte und wollte das Spiel zweimal mittendrin abbrechen, also aufgeben, wie im richtigen Leben auch. So kam es zum von Moreno (1959/2008, S. 81 f.) beschriebenen Kampf zwischen Protagonist und Leiterin.

Ich verlangte viel, indem ich F. aufforderte seinen inneren Konflikt Schritt für Schritt offenzulegen. Das Gruppenspiel brachte ihn an seine inneren Grenzen, in dem er darin Verhaltensweisen und Beziehungsverhalten zur Sprache brachte, die er bisher hinter die Schreibblockade verdrängt hatte. Im Spiel äußerte es sich als Wortfindungsstörung, als Ablenken von der Spielsituation und in diesen Phasen auch als Desinteresse der MitspielerInnen. Es war meine Aufgabe, den Protagonisten zum Themenkern zurückzuführen. Der erlösende Höhepunkt, nicht nur für F., sondern auch für seine Mitspielerinnen war, wie das spätere Sharing zutage brachte, als der Druck der Mutter, der sich als Sorge herausstellte, sich in Fürsorge wandelte und die Mutter durch das Halten der Hände und das in liebvolles In-die-Augen-Schauen 
Abb. 1 Teilnehmerin mit jahrelanger Schreibblockade sitzt bei unserer Schreibreise direkt nach der psychodramatischen Aufstellung unter einem Baum und schreibt

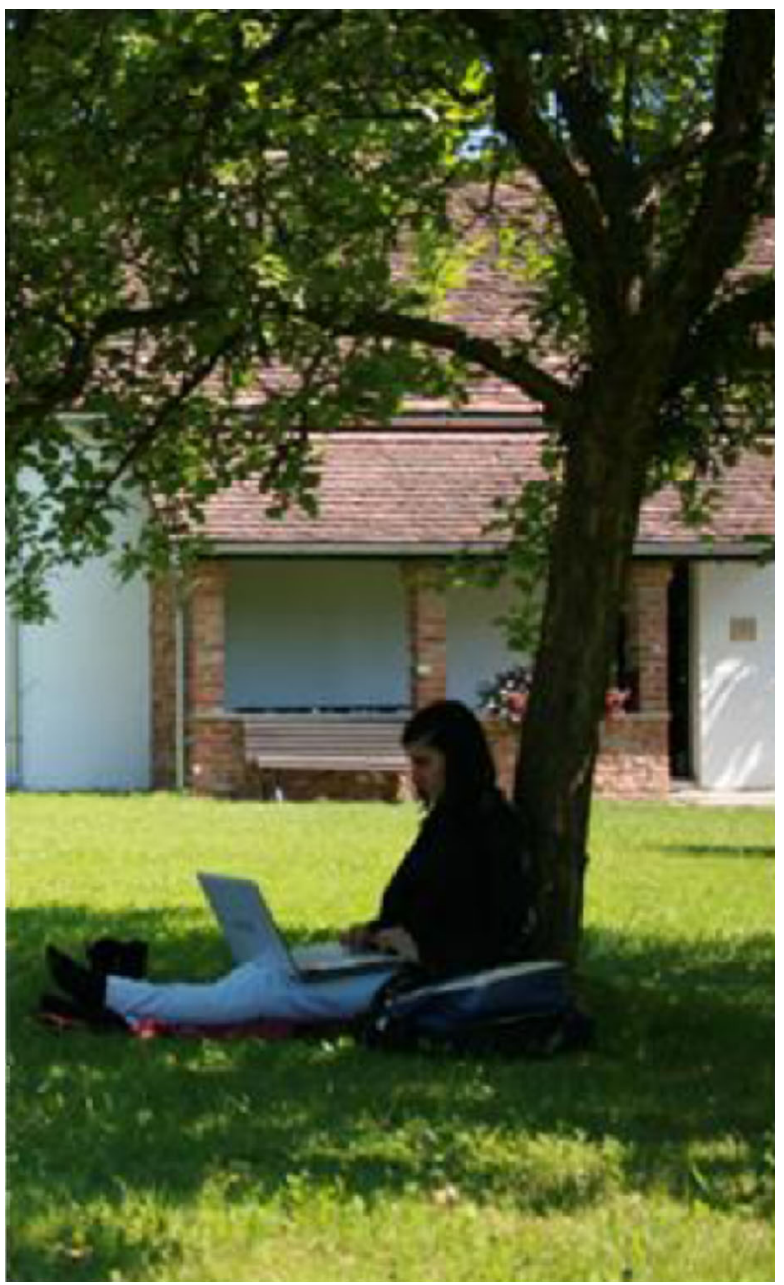

ihren Sohn mit neuer, von F. aufgenommener Energie in die Selbstverantwortung entließ.

Die fürsorglichen Anteile der Mutter bewirkten dann auch Integration der eigenen selbstfürsorgenden Anteile, was im Moment der Energieübertragung und dem Verlassen in die Selbstverantwortung eine fast unwirkliche Intensität annahm, die alle Mitspielenden als Kartharsis wahrnahmen. Und wirklich: Innerhalb der nächsten Stunde übernahmen alle Gruppenmitglieder die auf diese Art übertragende Selbstfürsorge und Eigenverantwortung: Sie begannen alle ernsthaft mit dem Schreiben (vgl. Abb. 1). In den Momenten, in denen sie mit der Aufgabenstellung überfordert waren, zogen sie während der Schreibreise die dort anwesenden Schreibberaterinnen hinzu und F. nahm sich auch nach der Schreibreise einige Beratungsstunden und konnte auf diese Weise seine Bachelorarbeit beginnen und dann auch, für ihn erstmalig, einen Text zeitgerecht einreichen. 


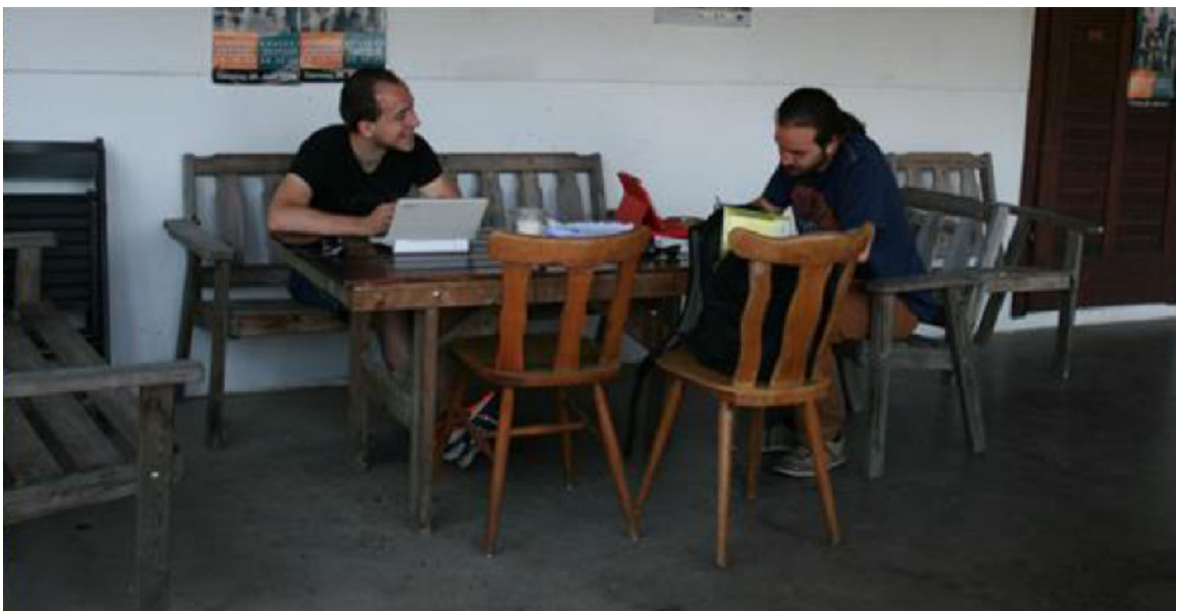

Abb. 2 Das Wissen, dass Schreibprobleme auch tiefere psychische Ursachen haben können, aber auch Schreibtechniken und gegenseitige Motivation helfen, verbindet die Schreibenden

Das Psychodrama als lebendige Methode orientiert sich zwar am Geschehen der Gruppe und am Erleben und Verhalten der GruppenteilnehmerInnen; der Inhalt einer Gruppensitzung ist aber nicht planbar (vgl. u. a. Hollenstein-Burtscher 2004, S. 193). So muss ich anmerken, dass auch ich als Leiterin selbst an meine Grenzen kam, als es darum ging, die Bewegung, die hinter der Prokrastination steckte, aufzudecken, ohne tieferliegende familiäre Beziehungskonflikte und Abhängigkeiten auf therapeutische Art und Weise zu thematisieren. In jedem Fall ist in einer Schreibwerkstatt eben so wenig tieferliegende therapeutische Arbeit zu betreiben wie in der Schreibberatung selbst. Sorgsam ausgebildete SchreibberaterInnen (z. B. am SchreibCenter der AAU Klagenfurt, am Schreibzentrum der PH Freiburg oder an der Ruhr-Universität Bochum, inhaltliche Anforderungen vgl. auch Grieshammer et al. 2012) wissen um die Möglichkeiten, aber auch um die Grenzen von Beratung und Werkstattarbeit Bescheid. Es darf keinesfalls zu einer entgrenzenden Erfahrung kommen, die eines therapeutisches Settings bedarf, und mit der ein Leiter oder eine Leiterin einer Schreibwerkstatt nicht mehr umzugehen weiß. Eine solche Entgrenzung kann in einer Schreibwerkstatt leicht passieren, auch schon beim Vorlesen autobiographischer Texte, wenn etwa TeilnehmerInnen Texte über eigene Gewaltoder Missbrauchserfahrung vortragen. Nur durch entsprechende Ausbildung ist das Know-how vorhanden, mit diesen Herausforderungen adäquat umzugehen.

Das auf der Schreibreise inszenierte Psychodrama empfanden die TeilnehmerInnen, wie man aus den schriftlichen Reflexionen entnehmen kann, als sehr hilfreich. Hier einige kurze schriftliche Rückmeldungen darauf:

„Den Einstieg durch die [Psychodrama-]Gruppe fand ich sehr gut“

„Diese Dinge werden doch eher selten in einem Kurs angesprochen und vermutlich trägt das Ambiente dieser Gegend dazu bei, dass offener darüber gesprochen werden kann als in einem Hörsaal der Universität." (Vgl. auch Abb. 2) 
„Wir setzen uns mit den Ursachen und Gründen von Schreibproblemen auseinander und arbeiten gemeinsam an Lösungen. Ich würde es als hands-on-Methode beschreiben und bin mir sicher, dass mir das Erfahrene auch in meiner Studienkarriere weiterhelfen wird."

„Außerdem geht es beim Schreiben nicht nur um die Sichtbarkeit, sondern darum, dass ich es gerne machen sollte und, wenn ich meiner selbst sicher bin, kann ich auch meine geschriebene Stimme erheben."

\section{Resümee}

Nach der psychodramatischen Aufarbeitung der Schreibprobleme berichteten die TeilnehmerInnen, wie leicht es war, ,sich fallen zu lassen, einfach drauf los zu schreiben und zu sagen: Es ist ja doch nur eine Rohfassung." Alle TeilnehmerInnen bewerteten diesen Kurse im Lehrveranstaltungsfeedback mit „,sehr gut“ und gaben an, durch diese Form nicht nur ihr Schreiben verbessert, sondern gleichzeitig sinnvolles gemeinsames Arbeiten erfahren und auch die Grenzen ihrer Möglichkeiten verschoben zu haben.

Zusammenfassend lässt sich sagen, dass es in jedem Fall eine Fülle an Möglichkeiten gibt, die Techniken aus Psychodrama mit den Techniken der Schreibwerkstatt produktiv miteinander zu kombinieren. Fundierte Ausbildungen in Schreibberatung und auch in Psychodrama, eventuell auch mit zwei WerkstattleiterInnen, die jeweils einen Teil abdecken, sind für diese herausfordernde Arbeit aber unerlässlich.

Acknowledgements Open access funding provided by University of Klagenfurt.

Open Access This article is distributed under the terms of the Creative Commons Attribution 4.0 International License (http://creativecommons.org/licenses/by/4.0/), which permits unrestricted use, distribution, and reproduction in any medium, provided you give appropriate credit to the original author(s) and the source, provide a link to the Creative Commons license, and indicate if changes were made.

\section{Literatur}

Bereiter, C. (1980). Development in Writing. In L. Gregg, \& E. Steinberg (Hrsg.), Cognitive processes in wirting (S. 73-93). Hillsdale, NJ: Erlbaum.

Bräuer, G. (1996). Warum schreiben? Schreiben in den USA: Aspekte, Verbindungen, Tendenzen. Frankfurt a.M., New York: Peter Lang.

Bräuer, G. (2006). Peter Elbows Konzept des freewriting als Paradigemwechsel in der amerikanischen Schreibpädagogik. In J. Berning et al. (Hrsg.), Schreiben im Kontext von Schule, Universität, Beruf und Lebensalltag (S. 11-28). Berlin: Lit-Verlag.

Elbow, P. (1973). Writing without teachers. London: Oxford Univ. Press.

Elbow, P. (1981). Writing with power. Techniques for mastering the writing process. New York: Oxford Univ. Press.

Girgensohn, K. (2007). Neue Wege zur Schlüsselqualifikation Schreiben. Autonome Schreibgruppen an der Hochschule. Wiesbaden: VS Verlag.

Girgensohn, K., \& Sennewald, N. (2012). Schreiben lehren, Schreiben lernen. Eine Einführung. Darmstadt: WBG.

Glindemann, B. (2001). Creative Writing in England, den USA und Deutschland. Kulturelle Hintergründe, literaturwissenschaftlicher Kontext, institutioneller Bezug. Frankfurt/M: Peter Lang.

Grieshammer, E., Liebetanz, F., Peters, N., \& Zegenhagen, J. (2012). Zukunftsmodell Schreibberatung. Eine Anleitung zur Begleitung von Schreibenden im Studium. Baltmannsweiler: Schneider. 
Hollenstein-Purtscher, A. (2004). Ablauf des Psychodramas. In J. Fürst, K. Ottomeyer, \& H. Pruckner (Hrsg.), Psychodrama-Therapie. Ein Handbuch. Wien: Facultas.

Keseling, G. (2004). Die Einsamkeit des Schreibers. Wie Schreibblockaden entstehen und erfolgreich bearbeitet werden können. Wiesbaden: VS Verlag für Sozialwissenschaften.

Kruse, O. (1993). Keine Angst vor dem leeren Blatt. Ohne Schreibblockaden durchs Studium. Frankfurt/New York: Campus.

Kruse, O. (2007). Schreibkompetenz und Studierfähigkeit. Mit welchen Schreibkompetenzen soll die Schule ihre Absolvent/inn/en ins Studium entlassen? In M. Becker-Mrotzek (Hrsg.), Texte schreiben. Kölner Beiträge zur Sprachdidaktik, (Reihe A, S. 117-143). Duisburg: Gilles \& Francke.

Mertlitsch, C. (2010). Starke Texte schreiben. Anregungen für Menschen im Arbeitsfeld Schule. Wien: BMUKK.

Mertlitsch, C. (2013). Die eigene Stimme finden. ide. Zeitschrift für den Deutschunterricht in Wissenschaft und Schule, (3), 80-87.

Moreno, J.L. (1959). Gruppenpsychotherapie und Psychodrama. Einleitung in die Theorie und Praxis. Stuttgart: Thieme.

Rose, M. (1984). Writer's block. The cognitive dimension. Carbondale: Southern Illinois University.

Ruhmann, G. (1996). Schreibblockaden und wie man sie überwindet. In K.D. Bünting et al. (Hrsg.), Schreiben im Studium. Ein Trainingsprogramm (S. 108-119). Berlin: Cornelsen Scriptor.

Soppa, P. (2001). Psychodrama. Ein Leitfaden. Wiesbaden: VS Verlag für Sozialwissenschaften.

Varga von Kibed, M. (2013). Drehbuchstrukturaufstellungen. https://www.youtube.com/watch?v=QE0XruFTkE. Zugegriffen: 1.6.2015

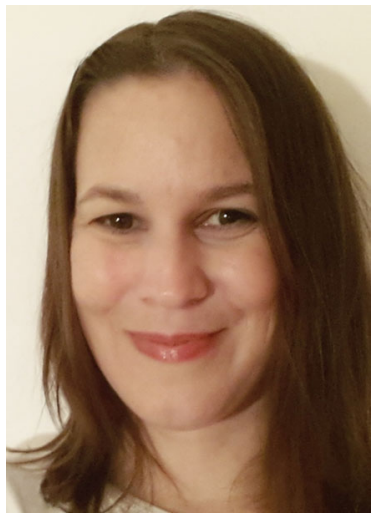

Carmen Mertlitsch geb. 1973, ist seit 2005 operative und stellv. wissenschaftliche Leiterin des SchreibCenters der Alpen-Adria-Universität, an dessen Aufbau sie maßgeblich beteiligt war. Sie studierte Germanistik, Psychologie, Philosophie und Angewandte Sprachwissenschaft an den Universitäten Wien und Klagenfurt, und besuchte einige Intensivseminare zu Psychodrama und Soziometrie u. a. bei Klaus Ottomeyer und Michael Wieser. Carmen Mertlitsch ist Gründungsmitglied der Gesellschaft Wissenschaftliches Schreiben Österreich (GEWISSS), Mitglied des Forum wissenschaftliches Schreiben Schweiz und Mitglied des Review-Kommitees der Zeitschrift Schreiben. 